\title{
Bandwidth Utilisation and Wavelength Re-Use in WDM Optical Burst-Switched Packet Networks
}

\author{
Michael Düser and Polina Bayvel \\ Optical Networks Group \\ Department of Electronic and Electrical Engineering \\ University College London \\ Torrington Place \\ London WCIE 7JE \\ United Kingdom \\ Tel: +44 207679 3843, Fax: +442073889325 \\ Email: pbayvel,mdueser@ee.ucl.ac.uk
}

Key words: Optical packet networks, burst switching

\begin{abstract}
Results describing the design trade-offs in bandwidth utilisation and wavelength re-use in optical burst-switched networks are reported. The effects of traffic statistics are analysed, and a set of bounds for the network design and lightpath set-up time for dynamic network control is derived. The round-trip time required for signalling is identified to be a key parameter in optimising the network performance.
\end{abstract}

\section{INTRODUCTION}

In future telecommunication networks traffic with different performance requirements will be merged in the same physical layer. The challenge in the design of future packet networks is, therefore, not only the increase in traffic volumes, but also the diversity of performance measures, such as packet delay, delay jitter and packet loss rate (PLR) for the different traffic types and dynamically varying network loads.

Although static wavelength-routed optical networks (WRONs) [1, 2] are relatively simple to design and operate, they are not easily adaptable to rapidly dynamically varying traffic, in sub-millisecond timescales. In 
contrast, optical burst-switched (OBS) networks [3-9] can accommodate dynamic traffic variations and differential quality of service (QoS) requirements by aggregating packets at the network edge to a burst, which is assigned to a free wavelength and sent into the network once pre-defined performance parameters have been exceeded. In this paper, an OBS architecture is described that allows to achieve deterministic latencies, together with a description of design parameters. New analysis and results are reported on the trade-offs in the design of edge delay, core-to-input bitrate ratio and the round trip time, which allow to predict bandwidth utilisation and dynamic wavelength allocation gain. These results can be used to define the bounds on the round-trip time required for the setting up of lightpaths to achieve a dynamic network control scheme.

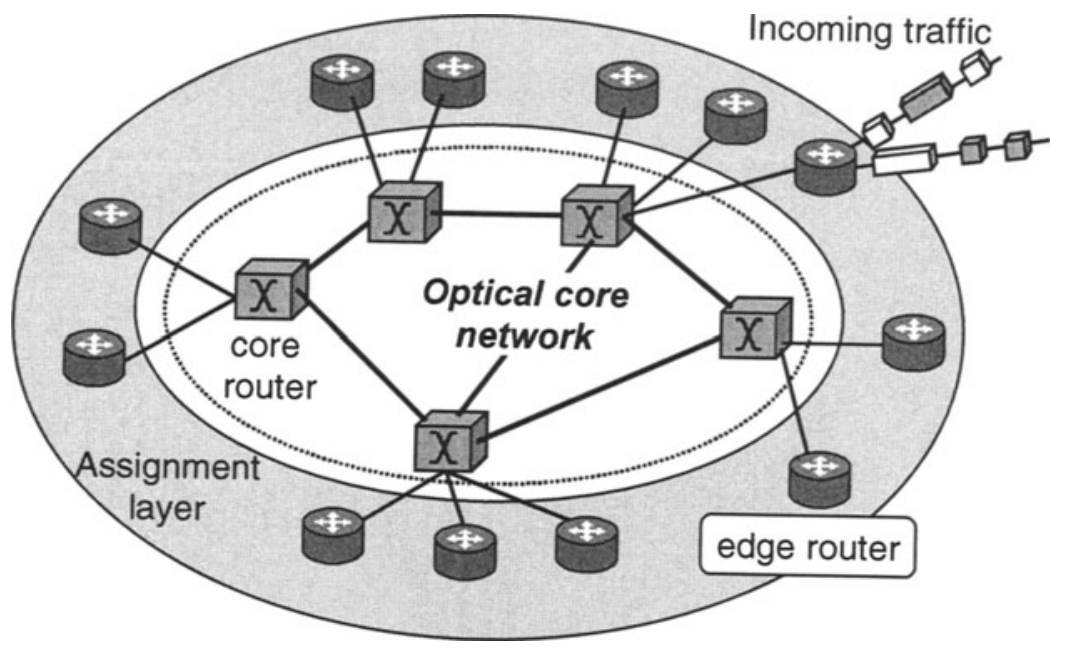

Fig. 1. Optical burst switching network architecture with electronic edge routers and optically transparent optical core

\section{OPTICAL NETWORK ARCHITECTURE AND TRAFFIC MODELLING}

The analysis in this work describes the network performance results presented in [7] in more detail and presents a detailed description of the assumptions made for the traffic modelling. The model is based on the OBS architecture shown in Figure 1, where electronic edge routers are connected to an optical core router. The optical core is assumed to be transparent by using dense wavelength-division multiplexing (DWDM) transport and 
passive routers to avoid the processing of header information and temporary buffering in the core routers. It is assumed that traffic at the edge consists of streams of packets, which are pre-sorted in the electronic edge-routers, according to their class of service (CoS) and destination into separate buffers as shown in Figure 2. Packets are not forwarded on a hop-by-hop basis as in IP networks, but are aggregated to flows, and dynamically assigned to an available wavelength, either when packets are dropped due to buffer overflow, or when a timeout signal indicates that time-critical packets have to be released to meet latency requirements. The incurred delay in the edge router, $t_{\text {edge }}$, is, thus, both deterministic and adjustable to meet the specific latency requirements of different traffic classes.

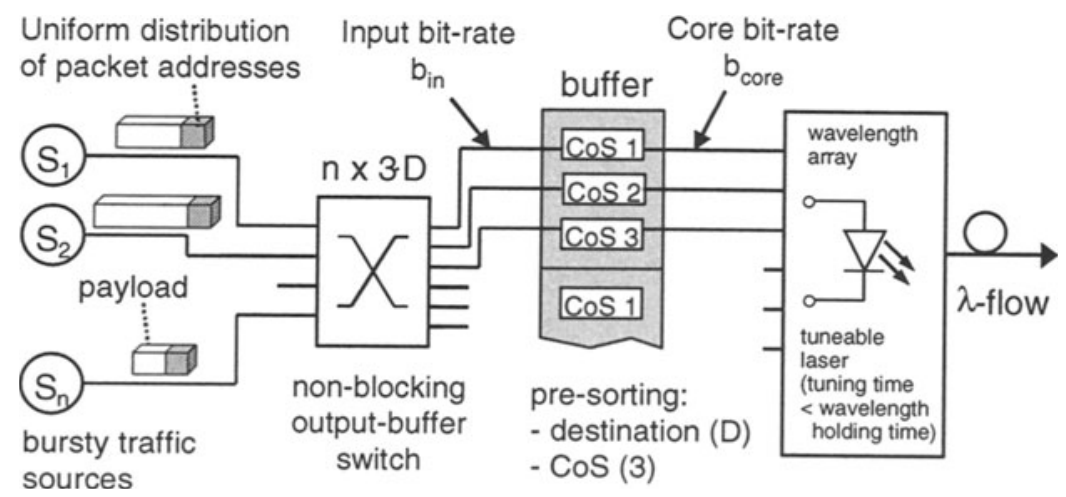

Fig. 2. Edge router model with electronic pre-sorting, non-blocking outputbuffered switch and tuneable wavelength section

The implementation of the burst aggregation functionality within the network edge router requires minor changes compared to the operation of electronic IP routers. The destination address is extracted from the packet header, and the output port determined from a table look-up. Using an output-queued switch to avoid loss from blocking of the switching matrix, the IP packet would be forwarded to the respective output queue. Although purely output-queued switches are difficult to realise, combined input-output queued (CIOQ) switches with moderate internal speed-up $(\leq 2)$ have been shown to achieve a throughput identical to output-queued switches [10].

\section{Comparison to other OBS schemes}

Optical burst switching combines the processing and buffering capabilities of electronics at the network edge with the advantage of optical DWDM transport and wavelength routing in the core. The exploitation of the advantages of both schemes allows to implement QoS differentiation in the core network. Guaranteed delays, delay jitter and PLR as QoS parameters 
have to be ensured even for dynamically variable traffic. A comparison with other OBS schemes, shown in Table 1, reveals that they will experience difficulties to ensure QoS guarantees.

Different schemes for the implementation of OBS networks have been suggested, but except the architecture discussed in this paper and the "Justenough-Time" (JET) scheme [8], without allowing for different CoS. Table 1 shows a summary of the functions of different implementations schemes with respect to admission, predictability of delay over the core network, burst delivery guarantees, and QoS considerations. The following abbreviations apply:

- Horizon: 'Horizon' scheduling algorithm

- TAG: tell-and-go

- JET: just-enough-time

- IBT: in-band-terminator

- RFD: reserved-fixed-duration

- JIT: just-in-time

- MPLS: multi-protocol label switching

Except the OBS architecture with admission control discussed in this paper, all schemes suffer from potential blocking of bursts in the optical core, resulting in packet loss and non-deterministic latencies.

Table 1. Comparison of different OBS implementation schemes

\begin{tabular}{llllll}
\hline Scheme & Admission & Delay & Delivery & QoS & Ref \\
\hline $\begin{array}{l}\text { Discussed } \\
\text { here }\end{array}$ & Limited & Deterministic & Guaranteed & Yes & {$[7]$} \\
Horizon & Full & Variable & $<100 \%$ & No & {$[4]$} \\
TAG & Full & Variable & $<100 \%$ & No & {$[6]$} \\
JET & Full & Deterministic & $<100 \%$ & Potentially & {$[6]$} \\
IBT & Similar to TAG & & & & {$[6]$} \\
RFD & Full & Variable & $<100 \%$ & No & {$[6]$} \\
JIT & Full & Deterministic & $<100 \%$ & No & {$[8]$} \\
MPLS-based & Limited & Deterministic & $<100 \%$ & Yes & {$[9]$} \\
\hline
\end{tabular}

\section{MODELLING RESULTS AND DISCUSSION}

A uniform distribution of packets addressed to other edge routers and negligible loss of packets in the output-queued switch are assumed, based on the assumption of very large buffers. The time duration before a burst is assigned to a free wavelength and released into the network is defined as the edge delay $t_{\text {edge. }}$. As the traffic statistics defines the build up of bursts, an earlier paper focused on analysing the effects of packet inter-arrival times and size statistics [7]. Simulations for traffic loads from 0.1 to 0.8 were carried out, showing that the worst case PLR was achieved for Pareto 
distributed packet lengths (400 bits - 320,000 bits) and Pareto distributed inter-arrival times, the best case was achieved with fixed length packets (400 bits) and Poisson inter-arrival statistics. It was shown that for the case of Pareto statistics the queues have to be emptied with at a faster rate to avoid buffer overflow. For the given simulation parameters this rate increase in the emptying of queues was approximately a factor of 2 .

Fig. 3. Source for traffic modelling with strictly alternating $\mathrm{ON}$ and $\mathrm{OFF}$ states

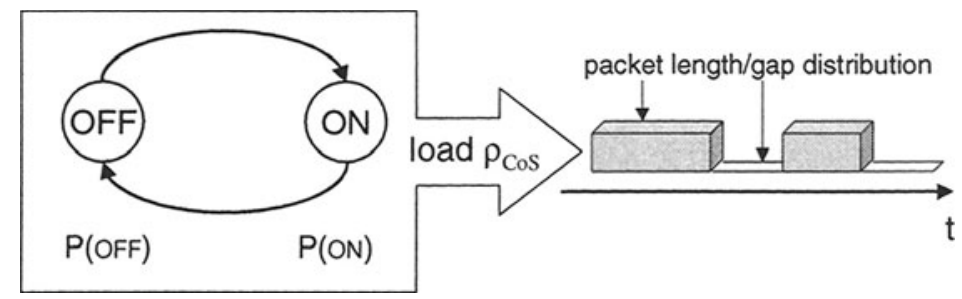

The incoming traffic is generated using ON-OFF sources as shown in Figure 3 with different probability density functions (PDF) for both the ONstate, $\mathrm{P}(\mathrm{ON})$, and the OFF-state, $\mathrm{P}(\mathrm{OFF})$ [11] to allow both variation of packet length and packet inter-arrival time. The source continuously alters between the ON and the OFF state, where different PDFs are applicable for both states to simulate a variety of traffic models.

The modelling of regular voice traffic can be described by Poisson interarrival time and exponential call holding times, but this model does not hold for the description of data traffic [12]. Although the correct model of data traffic is much under debate and strongly depends on the implemented protocol (e.g. IP, Ethernet), investigations report on the self-similar nature of traffic in deployed networks [13], showing that one possible implementation of this type of traffic is achieved when heavy-tailed Pareto distributions are multiplexed into the same queue. A Pareto distributed inter-arrival time $t$ is described as

$$
\mathrm{P}(\mathrm{t})=\frac{\alpha \cdot \mathrm{A}^{\alpha}}{\mathrm{t}^{\alpha-1}}
$$

where $1<\alpha<2, \mathrm{~A}>0$ and $\mathrm{t} \geq 0$. For detailed characteristics of a Pareto distribution, see [14]. For $1<\alpha<2$, the resulting statistical process at the output of the buffer is asymptotically second-order self-similar, where selfsimilarity is characterised by the Hurst parameter defined as [15]: 


$$
\mathrm{H}=\frac{3-\alpha}{2}
$$

so that $0.5<\mathrm{H}<1$. The importance of the self-similar traffic characteristics in the context of networking results from its influence on the PLR and the edge delay in buffers used for burst aggregation. The edge delay, $t_{\text {edge, }}$, is defined as the time that a burst spends in the buffer before being assigned to a free wavelength, so the queueing delay of packets entering the buffer is $\leq t_{\text {edge }}$.

To investigate the impact of traffic statistics on the queueing behaviour, simulations for both Poisson and Pareto inter-arrival statistics are shown to determine the distribution of the flow size, $\mathrm{L}_{\text {flow }}$, and the resulting PLR for a finite length buffer. Here, the results for two different traffic source characteristics are applied, and their impact on the flowsize and PLR as function of tedge is analysed for:

I) fixed length packet sizes, Poisson inter-arrival distribution

II) Pareto $(\alpha=1.5)$ packet length distribution, Pareto $(\alpha=1.5)$ interarrival time distribution

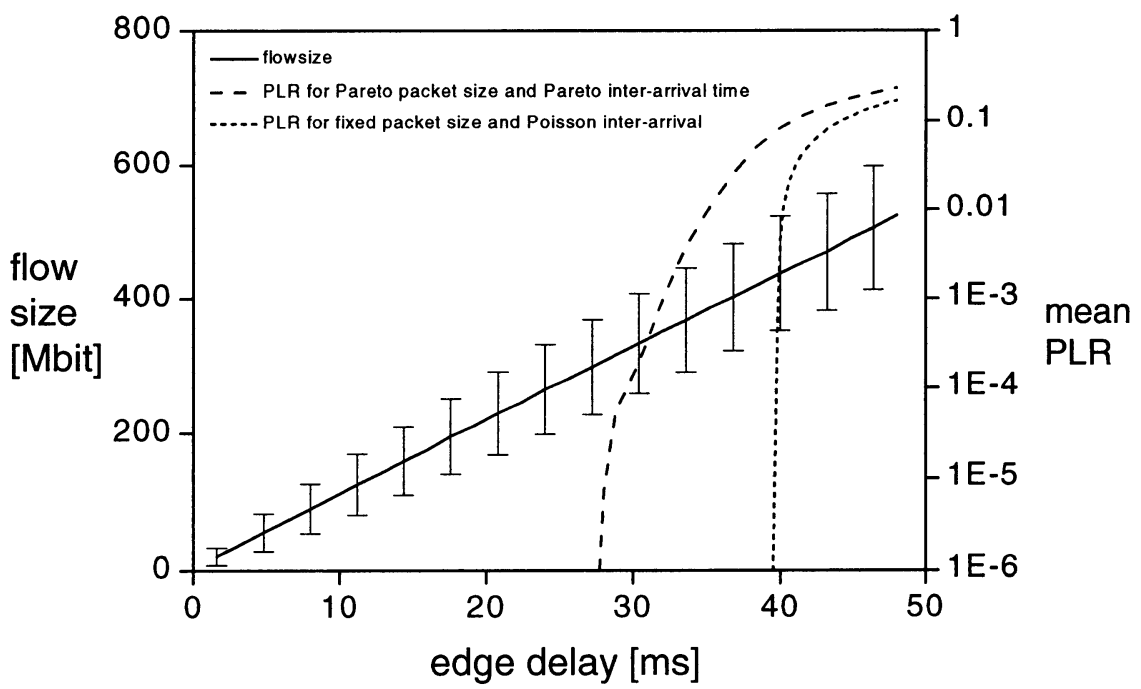

Fig. 4. Simulation results for flow size and PLR as a function of the edge delay for $B=400 \mathrm{Mbit}$ and a mean input bit-rate $b_{\text {in }}=10 \mathrm{~Gb} / \mathrm{s}$. Flow size (solid) and a mean PLR for fixed packet, Poisson inter-arrival (dotted) and Pareto packet size, Pareto inter-arrival (dashed) distribution 
From Figure 4 it can be seen that the mean flowsize increases linearly with $t_{\text {edge }}$ for both traffic models. Although the standard deviation of the flowsize is negligible in case I, a significant standard deviation is observed for case II of very bursty traffic, e.g. a standard deviation of 85.73 is observed for $t_{\text {edge }}=40 \mathrm{~ms}$. In the case of cbr traffic, the buffer is filled for a value of $t_{\text {edge }}=40 \mathrm{~ms}$, and a PLR $>0$ is expected for $t_{\text {edge }}>40 \mathrm{~ms}$. The impact of the very burst traffic on the related PLR is severe: Although in case I a significant PLR is observed $\left(>10^{-6}\right)$ for edge delays of approximately $40 \mathrm{~ms}$, i.e. nearly completely filled buffer, the same PLR is observed in case II for edge delays $<30 \mathrm{~ms}$.

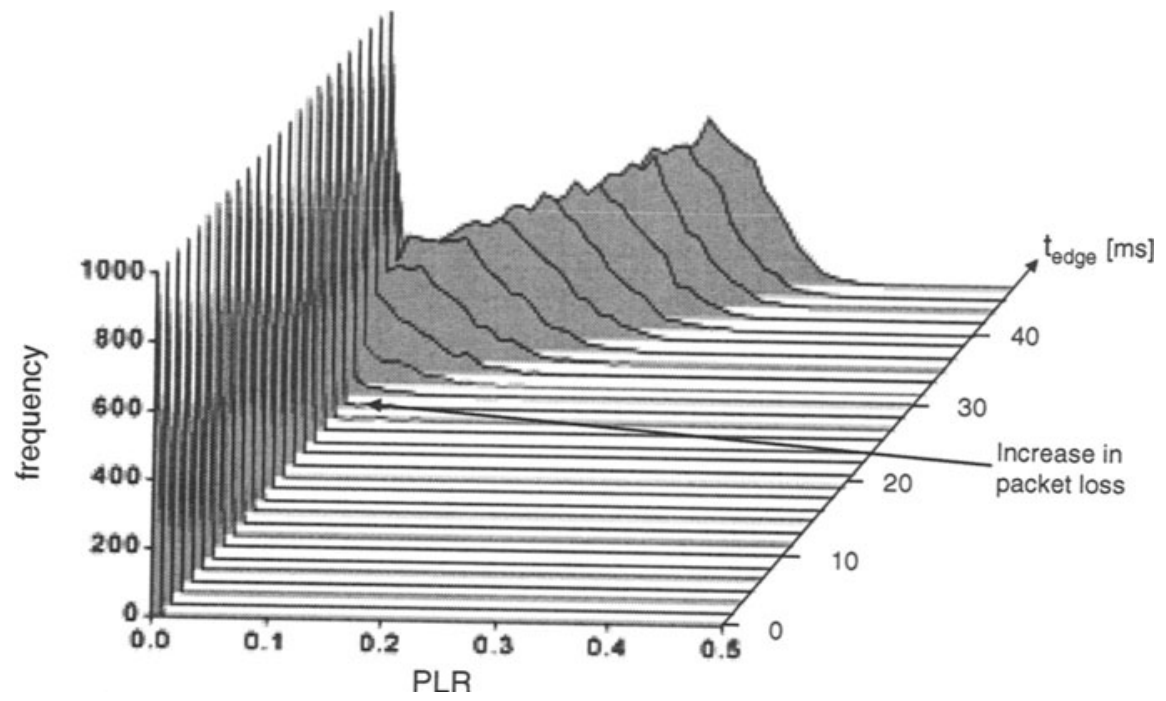

Fig. 5. Graph showing the PLR distribution as a function of edge delay. Significant deviations of the PLR from the mean is observed for Pareto traffic statistics $(\alpha=1.5)$ at the input for $t_{\text {edge }}>30 \mathrm{~ms}$.

Figure 5 shows the further analysis of the simulation results for the PLR, which reveal that significant deviations from the mean PLR can occur, resulting in occasionally excessive packet loss. For the implementation of QoS in OBS networks, therefore, not only the average PLR is to be taken into account, but also occasionally occurring packet loss.

In this analysis, the investigation of the effects of different statistics on the buffer overflow characteristics allows to predict and prevent excessive packet loss by introducing and adjusting threshold values, and, once a predefined threshold is exceeded, the burst will be assigned to the next available wavelength. As can be seen from Figure 4, an impairment of this scheme is 
that the burst aggregation time is limited (e.g. to $<30 \mathrm{~ms}$ in Figure 4 for selfsimilar traffic). Hence buffers are emptied at a higher rate, which increases the number of bursts in the core network at any given time.

Without anticipating results of the analysis in chapter 4 , it shall be noted at this place that long burst are beneficial for an efficient use of the network resources, especially to encourage the re-use of given wavelength paths. If, as shown above, bursty traffic entering edge routers reduces the maximum burst size, this will result in a decrease of the network performance.

Therefore, bursty traffic can be represented by the superposition of noise on the mean load, and continuous bit-rate (CBR) traffic represents the limiting case. For the buffer filling process, the decisive metric is the mean filling rate, or the mean flowsize, respectively. This is a valid approach since for identical traffic loads, but different traffic statistics, the mean values of the buffer filling process are identical, but the standard deviations vary significantly. Assuming that the buffer filling process and the mean value are of the main interest, and deviations can be neglected in the first instance, a traffic model independent on the traffic statistics can be applied by using CBR traffic. This allows for the analytical description of the proposed OBS scheme for all possible traffic statistics. In this work a continuous bit-rate (CBR) traffic model was, therefore, assumed. In this case, the flow size $\mathrm{L}_{\text {flow }}$ increases linearly with the edge delay

$$
\mathrm{L}_{\text {flow }}=\mathrm{t}_{\text {edge }} \cdot \mathrm{b}_{\text {in }}
$$

The wavelength holding time $t_{\mathrm{WHT}}$ denotes the period for which a given wavelength is assigned, typically in milliseconds

$$
\mathrm{t}_{\mathrm{WHT}}=\mathrm{t}_{\mathrm{RTT}}+\left(\mathrm{t}_{\text {edge }} / \mathrm{A}\right)
$$

where $A=b_{\text {core }} / b_{\text {in }}$ is the bit-rate ratio, the significance of this parameter is explained later together with Figs. 7 and 9. $t_{R T T}$ is the time required for the lightpath set-up, including the propagation delay. In these calculations a value of $5 \mathrm{~ms}$ was assumed, based on a network with a $1000 \mathrm{~km}$ diameter. It is assumed that this represents a propagation delay during which burst assembly takes place. This does not include the wavelength request processing at the control node. The equivalent bandwidth used for a path is defined as bandwidth-per-wavelength

$$
\mathrm{B} / \lambda=\mathrm{L}_{\text {flow }} / \mathrm{t}_{\mathrm{WHT}}
$$

For high resource utilisation in the physical layer it is important that a given lightpath is used as efficiently as possible, the bandwidth utilisation $\mathrm{U}$ can thus be defined as:

$$
\mathrm{U}=\frac{\mathrm{B} / \lambda}{\mathrm{b}_{\text {core }}}=\frac{\mathrm{t}_{\text {edge }}}{\mathrm{A} \cdot \mathrm{t}_{\mathrm{RTT}}+\mathrm{t}_{\text {edge }}}
$$


and is plotted in Figure 6 for $t_{\mathrm{RTT}}=5 \mathrm{~ms}$, and $b_{\text {core }}=12.5,40$ and $100 \mathrm{~Gb} / \mathrm{s}$ respectively. These values correspond to bit-rate ratios $\mathrm{A}=1.25,4$, and 10 for $b_{\text {in }}=10 \mathrm{~Gb} / \mathrm{s}$. It turns out that for the lowest core bit-rate, $12.5 \mathrm{~Gb} / \mathrm{s}$, the utilisation is maximum. This is due to the fact that the overhead caused by signalling for wavelength path set-up is minimum in this case, resulting in wavelength-holding times $t_{\mathrm{WHT}}$ in the range of tens of milliseconds, whereas $t_{\mathrm{RTT}}=5 \mathrm{~ms}$ was assumed to be constant. The utilisation is very low, however, for high bit-rates such as $100 \mathrm{~Gb} / \mathrm{s}$. Even for long edge delays in the range of $50 \mathrm{~ms}$, the utilisation is approximately $50 \%$ only.

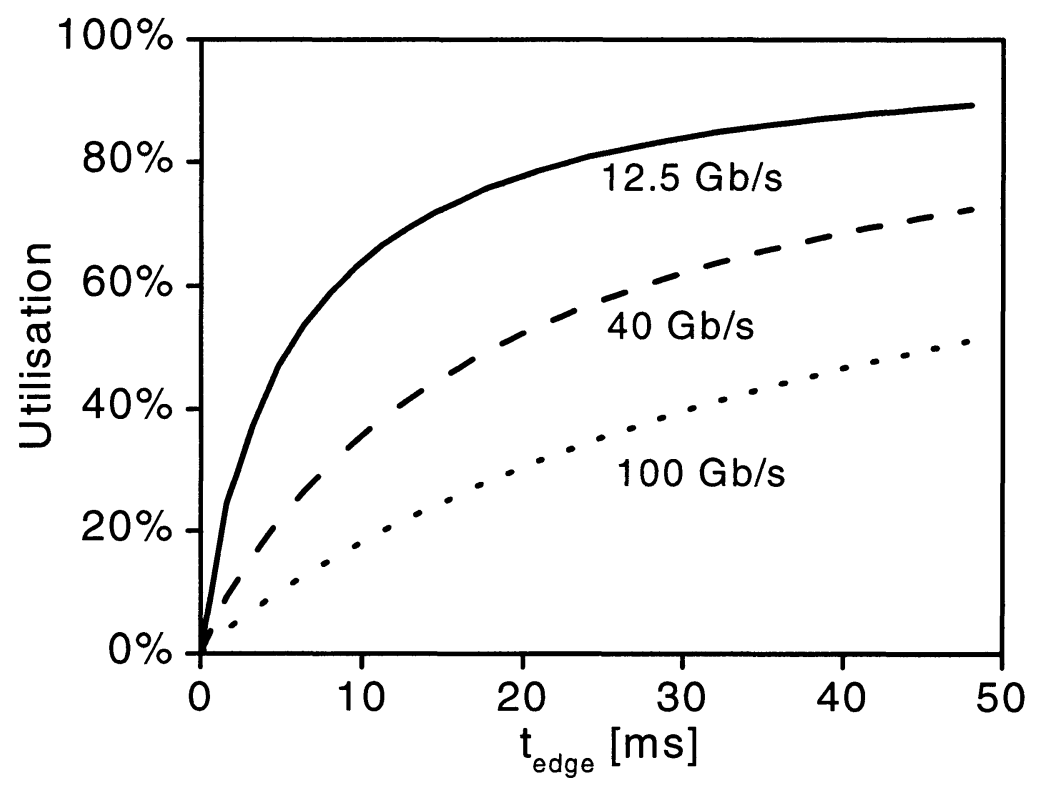

Fig. 6. Utilisation $U$ as a function of $t_{\text {edge }}$ for $t_{R T T}=5 \mathrm{~ms}, b_{i n}=10 \mathrm{~Gb} / \mathrm{s}$ and $b_{\text {core }}=12.5,40$, and $100 \mathrm{~Gb} / \mathrm{s}$

To investigate the influence of the edge delay and the bit-rate ratio A on the utilisation for a wider range of parameters, it is plotted in Figure 7 for 0 $\mathrm{ms} \leq \mathrm{t}_{\text {edge }} \leq 200 \mathrm{~ms}$, and $0 \leq \mathrm{A} \leq 100$. The range of values for $\mathrm{t}_{\text {edge }}$ and $\mathrm{L}_{\text {flow }}$ is based on previous simulation results of different packet size and packet inter-arrival times distributions, described in chapter 3 . 


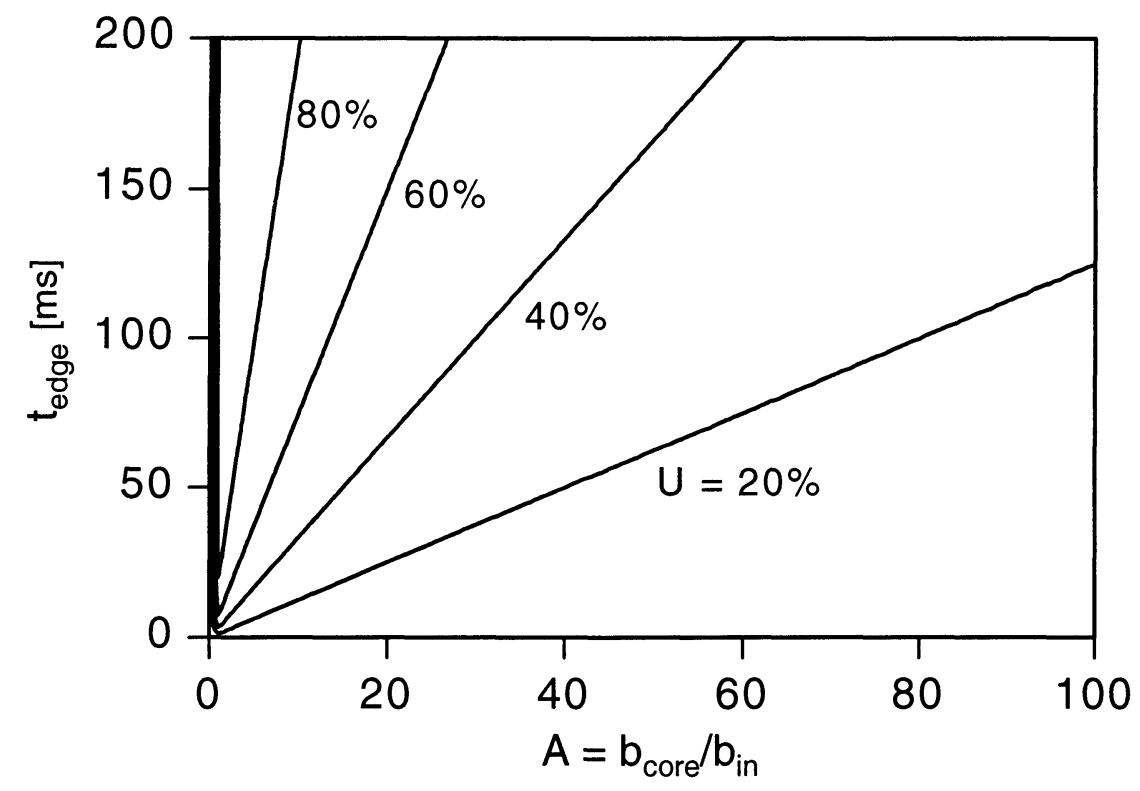

Fig. 7. Bandwidth utilisation, $U$, as a function of $t_{\text {edge }}$ and $A$ for a round-trip time $t_{\mathrm{RTT}}=5 \mathrm{~ms}$

As $b_{\text {core }} \gg b_{\text {in }}$ can be assumed for a high-speed optical core, $t_{\mathrm{WHT}}<<t_{\text {edge. }}$. In this case the time required to transmit a burst and, therefore, the time for which a given wavelength is used is much shorter than the edge delay. In the case of dynamic wavelength allocation an unused wavelength can be assigned to another edge router, and the resultant increase in the wavelength re-use is denoted by a wavelength re-use factor, RUF, defined as:

$$
\mathrm{RUF}=\frac{\mathrm{t}_{\text {edge }}}{\mathrm{t}_{\mathrm{WHT}}}=\frac{\mathrm{A} \cdot \mathrm{t}_{\text {edge }}}{\mathrm{A} \cdot \mathrm{t}_{\mathrm{RTT}}+\mathrm{t}_{\text {edge }}}=\mathrm{A} \cdot \mathrm{U}
$$

It is plotted in Figure 8 as a function of $t_{\text {edge }}$ for $t_{R T T}=5 \mathrm{~ms}, b_{\text {in }}=10 \mathrm{~Gb} / \mathrm{s}$ and $b_{\text {core }}=12.5,40$, and $100 \mathrm{~Gb} / \mathrm{s}$, respectively. 


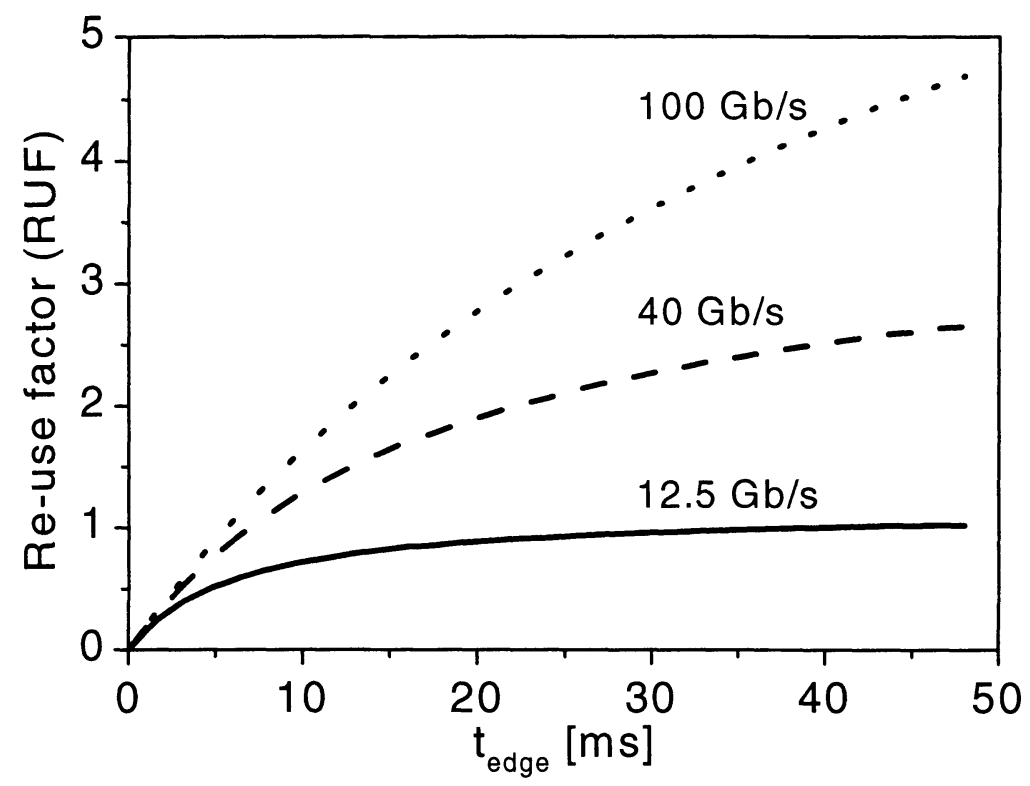

Fig. 8. Wavelength re-use factor RUF as a function of $t_{\text {edge }}$ for $t_{R T T}=5 \mathrm{~ms}$ and $b_{\text {core }}=12.5,40$, and $100 \mathrm{~Gb} / \mathrm{s}$

The wavelength re-use factor is plotted for a wider range of parameters in Figure 9 for $0 \mathrm{~ms} \leq \mathrm{t}_{\text {edge }} \leq 200 \mathrm{~ms}, 0 \leq \mathrm{A} \leq 100$, and $\mathrm{t}_{\mathrm{RTT}}=5 \mathrm{~ms}^{1}$.

From the above analysis it becomes evident that three parameters dominate the performance parameters of OBS networks:

- Edge delay $t_{\text {edge }}$

- Bit-rate ratio A

- Signalling overhead, characterised by the round-trip time $t_{R T T}$

Of particular importance is the bit-rate ratio A since in core networks the core bit-rate $b_{\text {core }}$ will be fixed in most cases. So, for given input bit-rate $b_{\text {in }}$, the bandwidth utilisation $U$ and the wavelength re-use RUF can be directly determined from Figs. 7 and 9 as a function of $t_{\text {edge }}$ and $t_{R T T}$.

\footnotetext{
${ }^{1}$ It should be noted that the wavelength re-use factor as defined here refers to the efficiency with which a given wavelength channel is used to satisfy other source-destination requests. It does not take into account the wavelength allocation algorithm or its efficiency in wavelength re-use to minimise the wavelength requirements.
} 


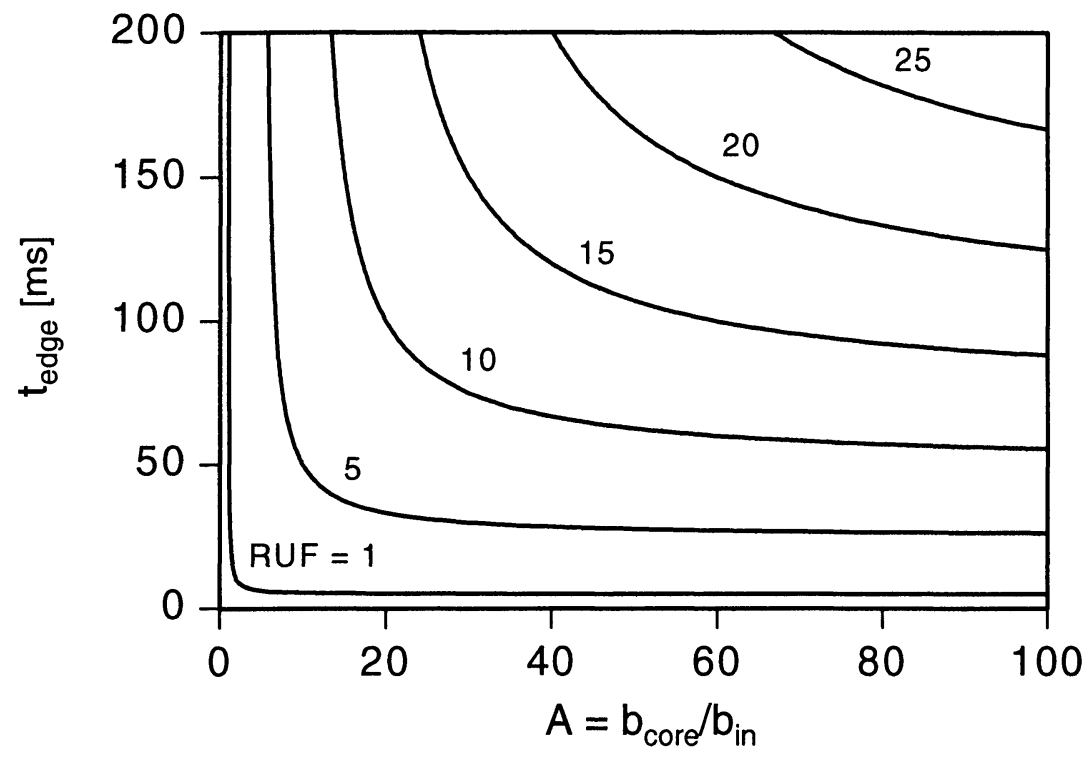

Fig. 9. Wavelength re-use factor, RUF for a round-trip time $t_{R T T}=5 \mathrm{~ms}$, as a function of $t_{\text {edge }}$ and $\mathrm{A}$

These results clearly show that with this network approach different types of traffic could be accommodated: low delays, required for time-critical types of traffic, are achievable, but with low values of utilisation, $U$, especially for large bit-rate ratios. It is possible to design a network with $U>$ $80 \%$ for $\mathrm{A}>10$ and delays $>50 \mathrm{~ms}$. From Figure 9 it can be seen that RUF reaches maximum values with both increasing $t_{\text {edge }}$ and $A$. For comparison, Figure 9 shows also the equivalent to the case of a statically wavelengthrouted optical network (WRON) where RUF $=1$. For values of RUF $<1$ the network would theoretically require more wavelengths than in a static WRON, and this represents the region of network instability where the total input load exceeds the network throughput.

The time to set up a lightpath is $t_{\mathrm{RTT}}$, required for signalling between edge routers and the network control element, either central or distributed. To ensure that RUF $>1$ as defined in (6),

$$
t_{\text {RTT }}<\frac{A-1}{A} \cdot t_{\text {edge }} \Leftrightarrow t_{R T T}<t_{\text {edge }} \text { for } A>>1
$$


It is important to note that in order to achieve efficient wavelength re-use, the lightpath set-up time must be as small as possible, and for a fixed $t_{\text {edge}}$, the upper bound in RUF is given by $\mathrm{RUF}_{\max }=\mathrm{A}$.

The proposed architecture can, therefore, accommodate traffic with wide range of delay requirements over the same network. Very delay sensitive traffic, such as voice, would only be queued for $10-20 \mathrm{~ms}$ before being assigned to a free wavelength with the penalty of a relatively low utilisation $(<50 \%)$ and re-use factor $(<5)$ as can be seen from Figures 7 and 9. A lower bound for the required edge delay is the round-trip time $t_{\text {RTT }}$. However, less delay sensitive traffic such as data can be routed over the network in parallel, but with longer edge delays allowing for a higher re-use of wavelengths than in the case of latency-sensitive traffic.

\section{SUMMARY}

New analysis of optical burst-switched networks is described which allows for the quantification of performance parameters key to network design. It was shown that for the limiting case of CBR traffic an analytical model for the edge router can be derived, and that this architecture allows to achieve a range of edge delays to satisfy the latency requirements of different traffic types. Bandwidth utilisation and wavelength re-use were introduced. These allow to quantify accurately the gain with dynamic wavelength allocation, which has the two-fold benefit of reducing wavelength requirements and enabling the network to respond to variable traffic demands. The time required for lightpath path set-up, $t_{R T T}$, is a lower bound on the achievable edge delays and must be minimised by fast RWA algorithms.

\section{Acknowledgements}

The authors would like to express their gratitude to Prof. S. Ferguson (Marconi Communications), Dr. D. Wischik (Cambridge), Dr. R. I. Killey (UCL), E. Kozlovski (UCL), A. Myers (UCL), and I. De Miguel (UCL, on leave from University of Valladolid, Spain) for invaluable comments. Financial support from Marconi Communications, UK EPSRC, and the Royal Society is gratefully acknowledged. 


\section{REFERENCES}

[1] S. Baroni, P. Bayvel, R.J. Gibbens, S.K. Korotky, "Analysis and design of resilient multifiber wavelength-routed optical transport networks," J. Lightwave Technologies 17, 743-758 (1999)

[2] S. Baroni, P. Bayvel, "Wavelength requirements in arbitrarily connected wavelengthrouted optical networks," J. Lightwave Technology 15, 242 - 251 (1997)

[3] J.S. Turner, "WDM Burst Switching for Petabit Data Networks," Tech. Dig. OFC 2000, paper WD2-1, 47 - 49 (2000)

[4] J.S. Turner, "Terabit Burst Switching," J. High-Speed Networks 8 (1), 3-16 (1999)

[5] C. Qiao, "Labeled Optical Burst Switching for IP-over-WDM Integration," IEEE Comm. Mag. 38 (9), 104 - 114 (2000)

[6] C. Qiao, M. Yoo, "Choices, Features and Issues in Optical Burst Switching," J. HighSpeed Networks 8 (1), 69 - 84

[7] M. Dueser, E. Kozlovski, R.I. Killey, P. Bayvel, "Design Trade-Offs in Optical Burst Switched Networks with Dynamic Wavelength Allocation," Tech. Dig. ECOC 2000, Vol. 2, paper Tu 4.1.4, 23 - 24 (2000)

[8] J. Y. Wei, J. L. Pastor, R. S. Ramamurthy, Y. Tsai, "Just-in-Time Optical Burst Switching for Multiwavelength Networks," IFIP Broadband '99, Hong Kong, Nov. $1999,339-352$

[9] S. Verma, H. Chaskar, R. Ravikanth, "Optical Burst Switching: A Viable Solution for Terabit IP Backbone," IEEE Network 14 (6), 48 - 53 (2000)

[10] S.-T. Chuang, A. Goel, N. McKeown, B. Prabhakar, "Matching Output Queueing with a Combined Input/Output-Queued Switch," IEEE J. on Select. Areas in Comms. 17, 1030 - 1039

[11] N. Likhanov, "Calculating Cell Loss Probabilities for ON-OFF Sources in Large Unbuffered Systems," IEEE Conf. on Communications (ICC'97), Vol. 2, 560 - 564 (1997)

[12] S. Keshav, "An Engineering Approach to Computer Networking: ATM Networks, the Internet, and the Telephone Network," Addison Wesley Longman Inc., Reading (MA), 1997

[13] L. Leland, M. S. Taqqu, W. Willinger, D. V. Wilson, "On the Self-similar Nature of Ethernet Traffic (Extended Version), "IEEE/ACM Trans. on Networking 2(1), 1 - 15 (1994)]

[14] V. Paxson, S. Floyd, "Wide Area Traffic: The Failure of Poisson Modeling," IEEE/ACM Trans. on Networking 3, 226 - 244 (1995)

[15] M. Parukelar, A. M. Makowski, "Tail probabilities for a multiplexer with self-similar traffic," Fifteenth Annual Joint Conference of the IEEE Computer Societies (INFOCOM'96), Vol. 3, 1452 - 1459 (1996) 